P74 (continued)

Outcome Measures and Analysis: The total expenditure on item level SSB purchases within the year 2011. A descriptive analysis (counts, frequencies) was conducted. The data was limited to descriptive analysis due to data limitations.

Results: SSB purchases differed between SNAP households and non-SNAP households. Non-SNAP households had a larger expenditure on SSBs than SNAP households. However, SSB constituted a somewhat larger percentage of total expenditures for SNAP households than non-SNAP households. For SNAP households, SSB items made up the majority of the top ten beverage purchases.

Conclusion and Implications: SSB consumption remains problematic in the U.S. and policies and education should be focused on reducing SSB consumption regardless of SNAP status. Rather than pointing to low-income households making poor choices, future research should investigate access to and availability of healthy food options in terms of distance to supermarkets and price and selection of available foods (corner stores).

Funding: None

\section{P75 Assessment of PSE Strategies in Arkansas SNAP-Ed Leads to Development of a Toolkit on PSE Implementation for Local Staff}

Brenda Wolford, MS, RD, brenda.wolford@altarum.org, Altarum Institute, 4 Milk Street, 3rd Floor, Portland, ME 4101; Brent Walker, MS, RD, Altarum Institute; Rosemary Rodibaugh, PhD, RD, LD, University of Arkansas; Sarah David, MBA, MM; Amy McClelland, RD, MS;

Katie Holland, MS, RD

Objective: To develop a comprehensive Policy, Systems and Environmental (PSE) change implementation toolkit and training after evaluating existing PSE strategies in Arkansas, the capacity of Family Consumer Science (FCS) agents to conduct PSE activities within their counties, and the training needs of local staff.

Theory, Prior Research, Rationale: USDA's Food and Nutrition Service stipulates that SNAP-Ed nutrition education classes must be delivered alongside comprehensive, multilevel interventions or community and public health approaches to improve nutrition. All states are required to deliver PSE strategies through multilevel or public health approaches to SNAP-Ed.

Description: The University of Arkansas Cooperative Extension Services (UACES) delivers nutrition education and physical activity promotion in 75 counties through SNAP-Ed. UACES contracted with Altarum Institute to help strengthen its approach to implementing and evaluating SNAP-Ed PSE approaches.

Evaluation: FCS agents participated in a survey and key informant interviews. A toolkit and training on PSE imple- mentation were developed based on findings from the survey and interviews.

Conclusions and Implications: The delivery of PSE strategies was already underway in many counties and the need for more training and support was identified. While agents had limited time to spend on SNAP-Ed PSE strategies, they realized the importance of partnerships and coordinated efforts to stretch their resources. Agents had a positive outlook on planning and implementing future PSE activities. A step-by-step toolkit was developed to assist agents with assessing, planning, monitoring and evaluating PSE strategies in their counties. A subsequent training on the toolkit was provided to agents by Altarum Institute with the goal of increasing their self-efficacy.

Funding: Supplemental Nutrition Assistance Program Education

\section{P76 Barriers to Fruit and Vegetable Consumption in the WIC Program: Counselor vs. Client Perceptions in a Midwestern City}

Katy Anderson, BS, Oklahoma State University; Stan Kogan, MPH, skogan@siouxfalls.org, South Dakota State University, 521 North Main Avenue, \#101, Sioux Falls, SD 57104

Objective: To compare WIC counselors' perceptions to their clients' self-reported barriers to fruit and vegetable consumption and cash value voucher (CVV) use in the Sioux Falls area.

Study Design, Setting, Participants, Intervention: Surveys were designed and validated with WIC and administered at the local WIC office. Surveys with clients were completed as interviews.

Outcome Measures and Analysis: Outcome measures included counselor and client perceptions toward increasing CVV use, fruit and vegetable consumption, and estimates of weekly food spending. Data was analyzed using Fisher's exact test and independent t-tests.

Results: There was a significant difference between counselor and client perceptions regarding adequate fruit and vegetable consumption ( $0 \%$ vs. $96 \%$; $p<0.001)$. Furthermore, there was a significant difference in counselor and client estimates of fruit and vegetable spending per week as a percentage of their food budget (10\% vs. $28 \%$; $\mathrm{p}=$ $0.004)$. On average, clients reported spending $\$ 10.62$ on fruits and vegetables per household member per week. Counselors and clients differed in their perceptions of improving CVV use. Most counselors believed that making it easier to use CVV while shopping would be the most impactful strategy, while most clients reported that cashiers not knowing how to check out with CVV was the biggest barrier. Counselors and clients agreed on strategies to improve fruit and vegetable consumption. The top strategy for both groups was learning to shop for produce on a budget. 
P76 (continued)

Conclusions and Implications: Counselors underestimated fruit and vegetable spending, but clients overestimated the adequacy of their fruit and vegetable consumption. Bringing those perceptions in line would likely help the WIC program better serve its clients in Sioux Falls.

Funding: None

\section{P77 Color Me Healthy for SNAP-Ed Supplemental Toolkit: A Comprehensive Approach to the Social-Ecological Model}

Jenelle Wass, MS, LDN, RD, jnwillia@ncsu.edu, North Carolina State University, 516 Brickhaven Drive, Raleigh, NC 27695; Gretchen Hofing, MPH, RD, North Carolina State University; Lindsay Goolsby, MS, LDN, RD;

Lindsey Haynes-Maslow, PhD, MHA;

Carolyn Dunn, PhD, RD

Objective: To enhance SNAP-Ed direct education messages with support for change on the interpersonal and organizational levels of the Social-Ecological Model (SEM).

Theory, Prior Research, Rationale: This project combines the Color Me Healthy for SNAP-Ed direct education program and the Nutrition and Physical Activity SelfAssessment for Child Care (NAP SACC) model, a research-tested intervention, to influence multiple levels of the SEM.

Description: North Carolina Extension educators receive training, on-going support, and an all-inclusive kit for direct education program implementation. Child care centers where Color Me Healthy for SNAP-Ed is delivered are engaged through a comprehensive toolkit for policy, systems, and environmental (PSE) change. This includes self-assessment, guided action planning, staff training, connections with local partners, and the provision of resources. Centers receive hands-on support throughout the process, creating personal connections that build trust and allow for greater center buy-in and a high completion rate, currently $82 \%$. Additionally, one-third of centers have participated for at least two years, fostering a longterm relationship.

Evaluation: The project encourages short, medium, and long term change on the individual and environmental levels, as defined by the SNAP-Ed Evaluation Framework. Administered twice in each annual cycle, NAP SACC, a reliable, valid, environmental self-assessment tool, was used to measure adoption and promotion of nutrition and physical activity supports, while surveys of parents and teachers captured childrens' behavior change.

Conclusions and Implications: The comprehensive application of the toolkit combined with direct education creates a multi-component, multi-level intervention that enhances the effectiveness and sustainability of PSE change at early child care centers.

Funding: USDA

\section{P78 Creating Healthy Behaviors Among Utah's SNAP-Ed Participants}

Casey Coombs, RD, casey.coombs@usu.edu, Utah State University Extension, SNAP-Ed, 8749 Old Main Hill, Logan, UT 84322; Mateja Savoie-Roskos, PhD, MPH, RD, Utah State University; Heidi LeBlanc, MS

Objective: To evaluate the curriculum's effectiveness by determining intention to change behaviors and actual behavior change of SNAP-Ed participants in Utah after receiving nutrition education based on the CREATE Curriculum. A convenience sample of SNAP-Ed participants was surveyed in 2014 and 2015. Participants were surveyed up to three points in time; after participation in one SNAP-Ed class $(n=6,825)$, after receiving at least 6 SNAP-Ed classes $(n=168)$, and 6 -months after receiving at least one SNAP-Ed class $(n=249)$. Participants were asked questions about dietary intake, physical activity, and cooking/mealtime habits. Wilcoxon signed rank test was used to compare nutrition-related behaviors in retrospective pre/post surveys. Participants reported an intention to improve all nutrition-related behaviors in pre-post surveys during the intervention $(\mathrm{p}<0.001)$. Participants also reported actually changing these behaviors six-months post-intervention $(p<0.001)$. After receiving six SNAP-Ed classes participants reported consuming more whole grains, fruits and vegetables and less saturated fats and processed food.

Description: The CREATE Curriculum is an innovative cooking-skills based curriculum developed by Utah's SNAP-Ed program aimed at increasing knowledge, skills, and self-efficacy of participants to help them make healthful nutrition-related decisions to prevent obesity and chronic diseases.

Results: The CREATE Curriculum may be an effective nutrition education tool for improving behaviors associated with a reduced risk of obesity among SNAP-Ed participants. SNAP-Ed programs nationwide should consider utilizing this curriculum in future programming. Future studies should follow SNAP-Ed participants over time to determine if behavior change is sustained.

Funding: Supplemental Nutrition Assistance Program Education

\section{P79 Dairy and Whole Grain Intake Differ by Food Security Status Among Indiana SNAP-Ed Eligible Adults}

Rebecca Rivera, MPH, rcusack@purdue.edu, Purdue University, 700 West State Street, West Lafayette, IN 47907; Melissa Maulding, MS, RD, Purdue University; Angela Abbott, MA, RD, CD; Qi Wang, MS; Heather EicherMiller, PhD

Objective: The Supplemental Nutrition Assistance Program-Education (SNAP-Ed) aims to improve the diet quality of low-income populations. Food insecurity 